\title{
The Effects of Longitudinal Vortices on Heat Transfer of Laminar Boundary Layers*
}

\author{
Kahoru TORII** and Jurandir Itizo YANAGIHARA**
}

\begin{abstract}
The isolated influence of longitudinal vortices "far enough" from a half-delta wing vortex generator on heat transfer of an otherwise laminar boundary layer was experimentally investigated. The heat transfer measurements were performed using a constant heat-flux surface with 96 embedded thermocouples. The vortex generator's height and angle of attack were varied to account for the influence of the vortex size, position and strength. A large increase in the heat transfer coefficient was found to be associated with the onset of the transition to turbulence rather than to vortical motion. The longitudinal vortices generated inside and outside the boundary layer showed different effects on heat transfer. When the transition did not occur, it was observed that the higher heat transfer coefficients were associated with the downwash region of the vortex, because of the local thinning of the boundary layer. The transition started in the upwash region of the vortex, where the instability was larger.
\end{abstract}

Key Words: Boundary Layer, Heat Transfer Enhancement, Longitudinal Vortices, Three-Dimensional Flow, Transition, Half-Delta Wing, Heat Transfer Measurements

\section{Introduction}

Longitudinal vortices embedded in laminar boundary layers are present in a variety of practical situations where the heat transfer is an important factor. Some examples are skew-induced vortices generated by blade or wing generators in compact heat exchangers, Taylor-Görtler vortices in curved surfaces or horseshoe vortices formed by protrusions from a surface. In many cases, a system of longitudinal vortices is purposely introduced to augment heat transfer rates. Heat transfer augmentation with small additional pressure loss is of economic importance for compact heat exchangers. The longitudinal vortex system is especially interesting because it is expected to increase the local heat transfer persistently over a long streamwise distance. This means that, once generated, the longitudinal vortex will disturb the entire velocity field and temperature field, expanding

\footnotetext{
* Received 19th September, 1988. Paper No. 88-2019A

** Faculty of Engineering, Yokohama National Univer. sity, 156 Tokiwadai, Hodogaya-ku, Yokohama, 240, Japan
}

its effect on heat transfer far downstream.

There have been many studies focusing on longitudinal vortices in various complex flow configurations, and some of them have presented heat transfer results. McCormack et al. ${ }^{(1)}$ investigated the effects of Taylor-Görtler vortices on heat transfer in laminar boundary layers. The local heat transfer rates were increased by as much as 150 percent over the flatplate values. This result may indicate a possible large effect of longitudinal vortices. Kottke ${ }^{(2)}$ carried out a similar work to investigate the influence of turbulence intensity and longitudinal vortices in concave plate boundary layers. He concluded that the longitudinal vortices are not the reason for sig. nificant turbulence-caused enhancement because high turbulence leads to a damping or destruction of longitudinal vortices. Recently, Schuz and Kottke(3) presented a paper on the effect of horseshoe vortices on heat and mass transfer. They offered information on the laminar-turbulent transition caused by longitudinal vortices and explained the influence of increasing the Reynolds number on the flow and the heat transfer.

More recently, some researchers have paid atten- 
tion to the use of longitudinal vortex generators to enhance heat transfer. The first results of heat transfer enhancement by vortex generators were reported by Edwards and Alker ${ }^{(4)}$, who investigated the effect of both counter-rotating and co-rotating vortices. The span-averaged results show that the enhanced coefficient was about 1.4 times as large as the unenhanced coefficient for the same plate location and flow conditions. The type of the boundary layer existing before the addition of the vortex generators is not given. Russel et al. ${ }^{(5)}$ carried out experiments with thermosensitive paints to determine the spanwise temperature distribution of alternating rows of closely spaced co-rotating vortex generators on a simulated plate-fin surface. Increases of up to 50 percent in heat transfer coefficient and 40 percent in pressure drop were reported. By the use of flow visualization and a yaw meter, the peak heat transfer locations were found to be associated with the downwash region between vortices. Fiebig et al. $^{(6)}$ reported an experimental work in which the local heat transfer and friction coefficients for channels with various kinds of wing-type vortex generators were measured. The span-averaged Colburn-factor enhancement ranged from 1 to 2 with modest increases in friction coefficients. Turk and Junkhan ${ }^{(7)}$ conducted similar work on blade-type vortex generators in flat plates, with an emphasis on the relationship between the geometry and the augmentation of the local and overall coefficients. The span-averaged heat transfer data revealed an increase of up to 150 percent in comparison with the otherwise laminar condition. None of these works, however, presents quantitative flow field informations enabling us to obtain correlations between the vortex dynamics and the heat transfer characteristics.

The work of Eibeck and Eaton ${ }^{(8) \sim(10)}$ examined in detail the isolated influence on heat transfer of a longitudinal vortex of various strengths embedded in a turbulent boundary layer. The provision of the spatially resolved heat transfer data coupled with three-component velocity measurements allowed the association of the local heat transfer behavior with the specific features of the vortex. Their results show that the longitudinal vortex imposes local modifications on the heat transfer coefficient through the distortion of the mean flow field rather than by modifying the turbulence field or by means of the larger skin friction magnitude caused by spanwise flow. The validity of the "law of the wall" in each spanwise station indicated that the flows seemed to be dominated by two-dimensional mechanisms near thewall. They also observed that the influence of longitudinal vortices on heat transfer was strongly persistent, with the circulation of the vortex decaying slowly. The measured averaged heat-transfer augmentation was modest when compared to the two-dimensional flat-plate value. Detailed mean flow and turbulence measurements for a similar flow can be found in the works of Shabaka et al. ${ }^{(11)}$, Westphal et al. ${ }^{(12)}$ and Mehta and Cantwell(13).

Pauley and Eaton ${ }^{(14)}$ extended the above-mentioned work to include the case of a pair of vortices. They observed a broad region of enhanced heat transfer between the vortices, leading to a substantial increase in the spanwise averaged heat transfer. They found that a pair of longitudinal vortices induces greater distortion in the boundary layer structure so that a strong correlation exists between the turbulence intensity near the wall and the local heat transfer coefficient.

In summary, although a considerable amount of work has been done to help us understand the fluiddynamic behavior of flows dominated by longitudinal vortices, its heat transfer aspects are not adequately known. In the case of laminar boundary layers, there is a lack of heat transfer results associated with fluid-dynamic information enabling us to understand the mechanism by which the enhancement of heat transfer is achieved.

The objective of the present work was to determine the influence of the strength, size and position of a single longitudinal vortex on the heat transfer of an otherwise laminar boundary layer "far enough" from the vortex generator. This was accomplished by varying the free-stream velocity and the vortex generator height and angle of attack during the experiments.

\section{Nomenclature}

$b$ : Width of 50 percent peak Stanton number region

$e$ : Shift of Stanton number distribution from centerline

$H:$ Vortex generator height

Pr: Prandtl number

Re: Reynolds number

sh: Relative shift

$S t:$ Stanton number

$S t_{0}:$ Stanton number for the laminar two-dimensional flow

$U:$ Free-stream velocity

$x$ : Spatial coordinate, streamwise direction

$x_{0}$ : Unheated starting length

$y$ : Spatial coordinate, normal direction

$z$ : Spatial coordinate, spanwise direction

$\alpha$ : Generator's angle of attack

$\delta:$ Velocity boundary layer thickness 


\section{Experimental Apparatus and Procedure}

The test facility consisted of a low-speed, opencircuit, zero-pressure grandient wind tunnel, as show in Fig. 1. The air was delivered by a controlled blower and passed through a turbulence management section. A nozzle with a contraction ratio of $7: 1$ connected the wind tunnel to the $260 \mathrm{~mm} \times 300 \mathrm{~mm}$ rectangular test section. The nominal free-stream velocity at the inlet plane was $3 \mathrm{~m} / \mathrm{s}$, with a free-stream turbulence intensity lower than 0.3 percent.

A single vortex was generated at the centerline of the laminar boundary layer by means of a half-delta wing and then passed over a constant heat-flux heat transfer surface (Fig. 2). A stainless-steel heated surface $(260 \times 1143 \times 0.03 \mathrm{~mm})$ was set up at the upperside wall of the test section to avoid the influence of natural convection. The back face of the heater was insulated by a sheet of balsa wood placed on an acrylic plate covered with expanded-styrol insulation. The conduction losses were evaluated to be 10 percent of the total heat generated, and the radiation losses were calculated by assuming an emissivity of 0.15 . The electrical power dissipated in the heated metal sheet was evaluated by measuring the voltage drop across the surface and the current supplied.

The distributions of heat transfer coefficients in both spanwise and streamwise directions were based

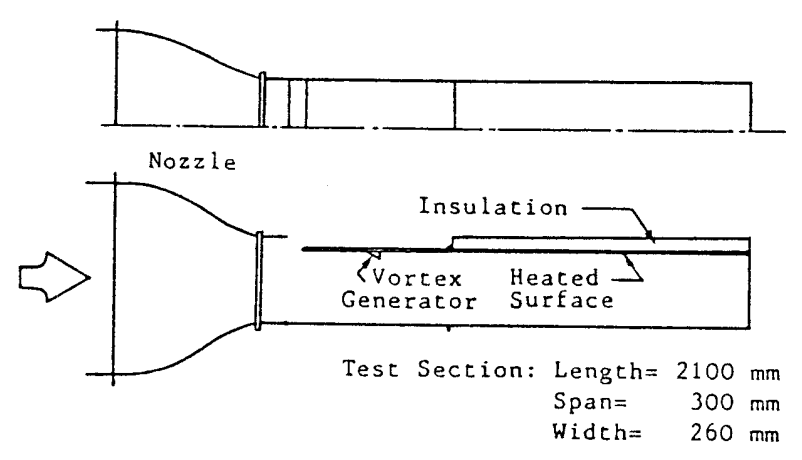

Fig. 1 Schematic view of the wind tunnel

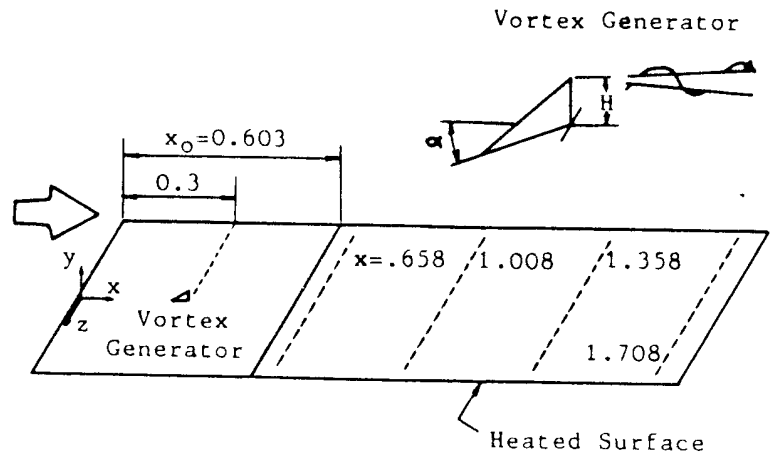

Fig. 2 Schematic view of the test surface on temperature measurements made by 96 fine thermocouples attached to the back of the heat transfer surface. Eighty-four cooper-constantan thermocouples $(0.06 \mathrm{~mm}$ in diameter) were arranged along the surface in four spanwise rows of 21 thermocouples each. Twelve additional thermocouples were distributed along the centerline, and seven thermocouples were positioned at seven different centerline positions at the back of the acrylic wall. The spacing of the thermocouples was $10 \mathrm{~mm}$ in the spanwise direction and $70 \mathrm{~mm}$ in the streamwise direction.

The uncertainty of the Stanton number was estimated by combining uncertainties in the measurements of the local temperature and the power supplied and in the corrections applied for the rediation and conduction losses ${ }^{(15)}$. The uncertainty in the temperature measurement was $0.5^{\circ} \mathrm{C}$, considering some possible errors associated with the A-D conversion, the measurement circuit, and the local temperature drop at the point of attachment of the thermocouple to the stainless steel. The uncertainty in the power supplied was estimated to be 2 percent, based on the accuracy of the instruments and fluctations in the power measurements during the data acquisition. The correction for the conduction and radiation losses was approximately 20 percent of the power supplied, with local variations. The uncertainty of this correction was the main contribution toward the uncertainty of the heat transfer coefficient. The resulted uncertainty of the Stanton number was about 10 percent, varying with the experimental conditions.

In fact, the heat transfer results are presented in nondimensional form as the Stanton number ratio $\left(S t / S t_{0}\right)$, normalized by the two-dimensional heat transfer data taken at the same point and submitted to the same data reduction procedure. The normalizing process compensates for some contributions of the measurement errors, mainly the bias error. Therefore, the uncertainty of the normalized Stanton number should be substantially lower than 10 percent.

The adequacy of the experimental apparatus was verified by taking the velocity profiles in various spanwise positions and by comparing the heat transfer data with theoretical expressions. The velocity profiles agreed well with the Blasius profile. The heat transfer data showed good two-dimensionality and agreed well with the following expression given by Rubesin et al. ${ }^{(16)}$ :

$$
S t * \operatorname{Pr}^{2 / 3}=0.460 * \operatorname{Re}^{-1 / 2} *\left(1-x_{0} / x\right)^{-1 / 3} \text {. }
$$

The longitudinal vortex was generated by six different half-delta wings whose heights $(H)$ were 3 , $4.5,6,10,15$ and $30 \mathrm{~mm}$. The generators were positioned $300 \mathrm{~mm}$ from the leading edge, where the boundary layer thickness $(\delta)$ was about $6 \mathrm{~mm}$, depending on the 
velocity. The wing sweep angle was fixed at $68^{\circ}$ and the angle of attack $(\alpha)$ was varied from $4^{\circ}$ to $25^{\circ}$. The free-stream velocity was varied from 2.61 to $2.96 \mathrm{~m} / \mathrm{s}$ to demonstrate the remarkable influence of slight variations of $H / \delta$.

\section{Results}

\section{1 Influence of the angle of attack}

At first, for the nearly constant free-stream veloc. ity of 2.92 to $2.98 \mathrm{~m} / \mathrm{s}$, the angle of attack was varied from $5^{\circ}$ to $25^{\circ}$ with a step of $\Delta \alpha=5^{\circ}$, using a vortex generator whose height was equal to the boundary layer thickness. Figure 3 represents the axial development of the Stanton number ratio distribution (St/ $\left.S t_{0}\right)$ for the five cases mentioned above. The peak Stanton number ratio for $\alpha=10^{\circ}$ to $25^{\circ}$ increased from 1.1 in the first row $(x=0.658 \mathrm{~m})$ to 2.4 in the fourth row $(x=1.708 \mathrm{~m})$, which means a considerable improvement of the heat transfer coefficient. It is worth noting that the enhanced region spread as the flow moved downstream and the point of the peak Stanton number shifted slightly in its spanwise position to the upwash side of the vortex. This general behavior was the same for the four cases considered, $\alpha=10^{\circ}$ to $25^{\circ}$. For $\alpha=5^{\circ}$, however, only a very small change in the heat transfer coefficient was observed. After some preliminary measurements, it was observed that this abrupt change between $\alpha=5^{\circ}$ and $10^{\circ}$ occurred for a specific value of $\alpha$ and was strongly related to the free-stream velocity. Figure 4 shows the axial development of the spanwise distribution of $S t /$ $S t_{0}$ for $\alpha=4.0^{\circ}, 4.5^{\circ}, 5.0^{\circ}, 5.5^{\circ}$ and $10^{\circ}$. From this figure, it is possible to observe a clear improvement of heat transfer even with a slight increase of the angle of attack. The Stanton number distribution along the centerline for these five cases is presented in Fig. 5, as a function of the local Reynolds number. The results depart from the laminar curve at a certain streamwise position. This position, which separates the region where the augumentation rate is large from that where it is small, moves upstream as the angle of attack increases. Since the transition to a turbulent boundary layer develops beyond the stage described by the classical two-dimensional linear stability theory when three-dimensional disturbances are present ${ }^{(17)}$, it is possible to consider that what occurs here is the onset of transition from laminar to turbulent regime. Consequently, the effective thermal diffusivity is increased. As the angle of attack is increased, the circulation of the vortex which arises from the generator becomes larger and hastenes the occurrence of transition.

\subsection{Influence of free-stream velocity}

In this series, the free-stream velocity was slight.

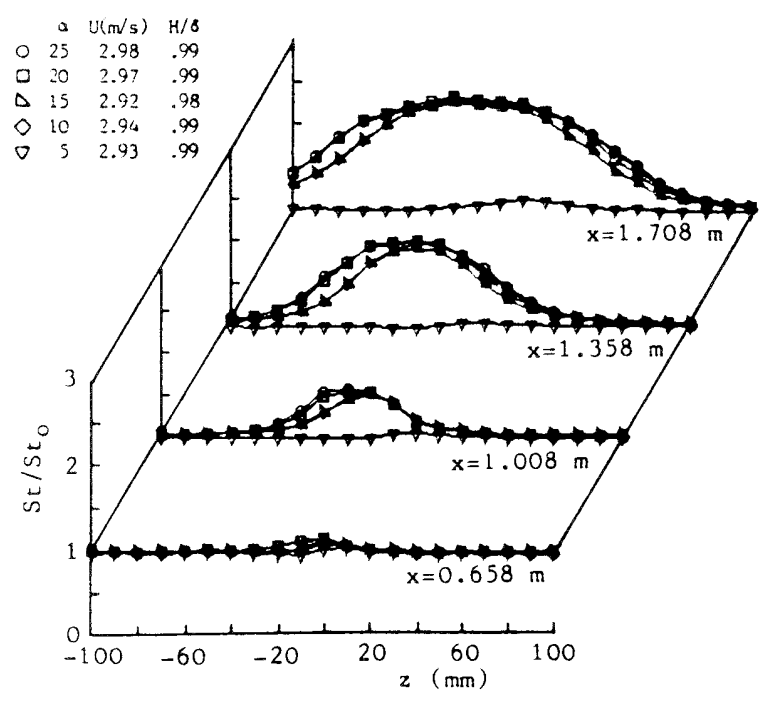

Fig. 3 Axial development of spanwise Stanton number ratio distribution (parameter: angle of attack)

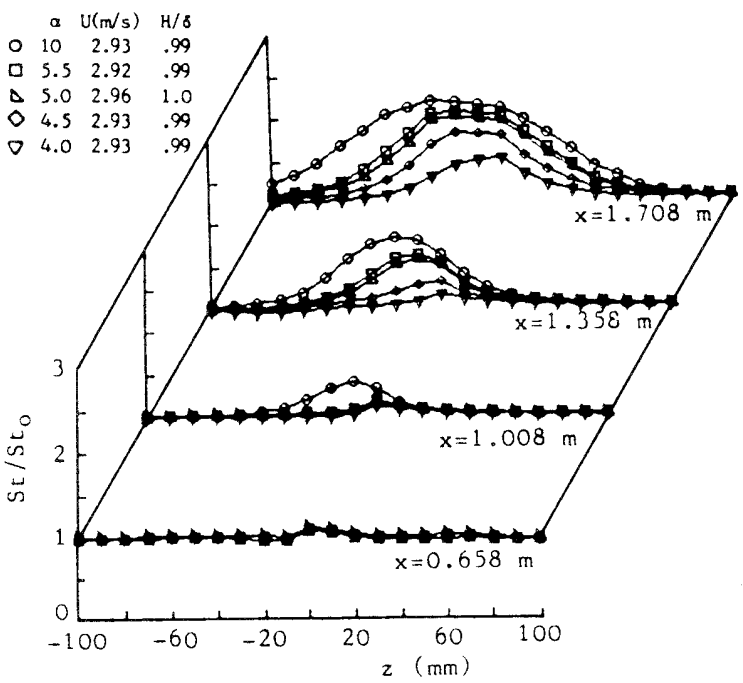

Fig. 4 Axial development of spanwise Stanton number ratio distribution (parameter: angle of attack)

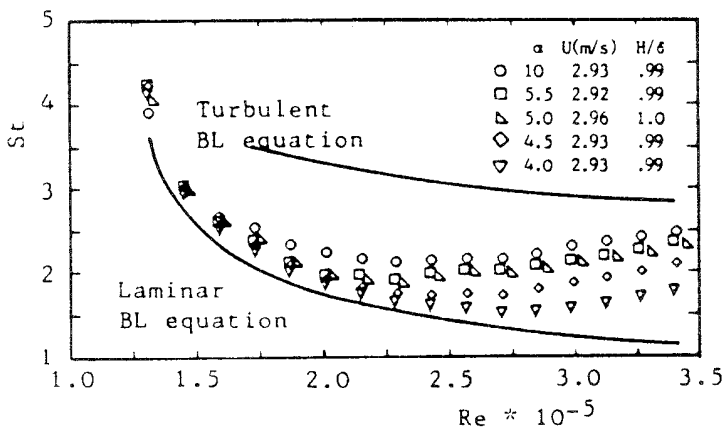

Fig. 5 Stanton number distribution along the center line for the five different angles of attack 
ly varied from 2.61 to $2.96 \mathrm{~m} / \mathrm{s}$. The same vortex generator was used for the four measurements $(H=6$ $\mathrm{mm}$ and $\alpha=5^{\circ}$ ). The results, shown in Fig. 6 , reveal the remarkable influence of the free-stream velocity and, consequently, of a slight variation of $H / \delta$. The augmentation rate was found to grow with increasing the velocity. Shown in Fig. 7 is the axial variation of Stanton number along the centerline which corresponds to the peak values for the four measurements in Fig. 6. Even for a slight increase of $0.35 \mathrm{~m} / \mathrm{s}$ in the velocity (or 0.06 in $H / \delta$ ), the position of transition moved $0.6 \mathrm{~m}$ upstream and the Stanton number ratio for the fourth row increased from near 1 to about 2 unities.

During the execution of this experimental series, the streamwise velocity fluctuation inside the boundary layer at a position $35 \mathrm{~mm}$ downstream of the fourth row of thermocouples was measured using a single hot-wire anemometer. In this case, although the flow was three dimensional, its streamwise component

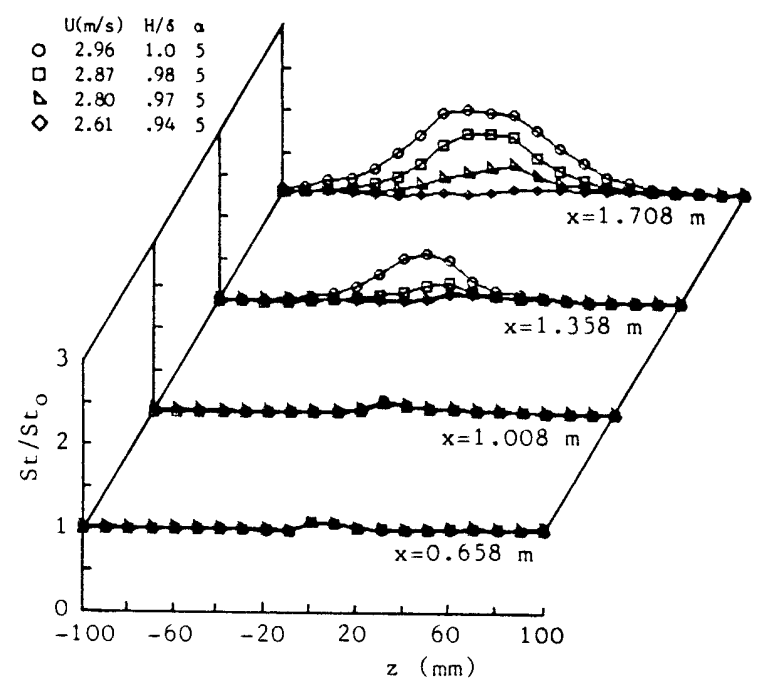

Fig. 6 Axial development of spanwise Stanton number ratio distribution (parameter : free-stream velocity)

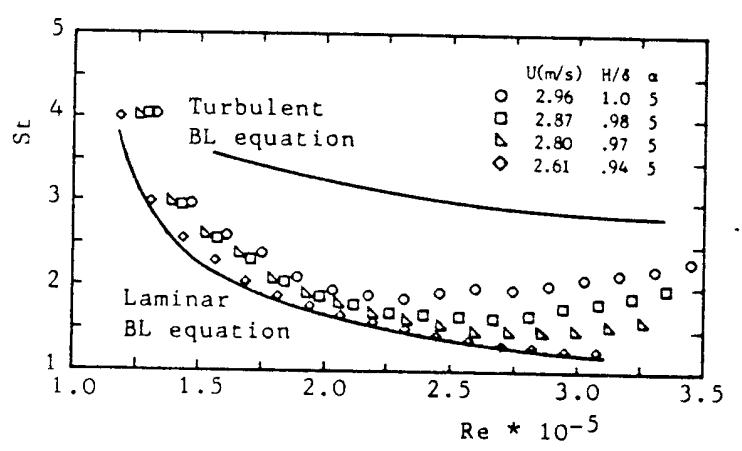

Fig. 7 Stanton number distribution along the centerline for the four different free-stream velocities was predominant. Therefore, the measurement of the turbulence intensity in the $x$ direction was sufficient to characterize the departure from the laminar regime. The results showed a change from nearly 0.5 percent for the two-dimensional case to a peak value of nearly 20 percent for $U=2.80 \mathrm{~m} / \mathrm{s}$ (Fig. 8). The spanwise variation of the turbulence intensity agreed quite well with the corresponding Stanton number ratio distribution at the same axial location. From the these measurements, it is possible to conclude that the visible augmentation in heat transfer was caused by the onset of transition to turbulent regime.

The isolated effect of the vortical motion on heat transfer of a laminar boundary layer is shown in Fig. 9. In this case, where the free-stream velocity is 2.61 $\mathrm{m} / \mathrm{s}$, no apparent transition occurs. The position of the peak Stanton number corresponds clearly to the downwash region, where the boundary layer becomes thinner. On the other hand, the minimum Stanton number is found to be associated with the upwash region of the vortex.

\section{3 Influence of height}

The influence of the vortex size, strength and position on the flow behavior and the heat transfer was studied by varying the generator height. The basic angle of attack was $15^{\circ}$ and the height ranged from 3 to $30 \mathrm{~mm}$, corresponding to $H / \delta=0.5$ to 5 . This

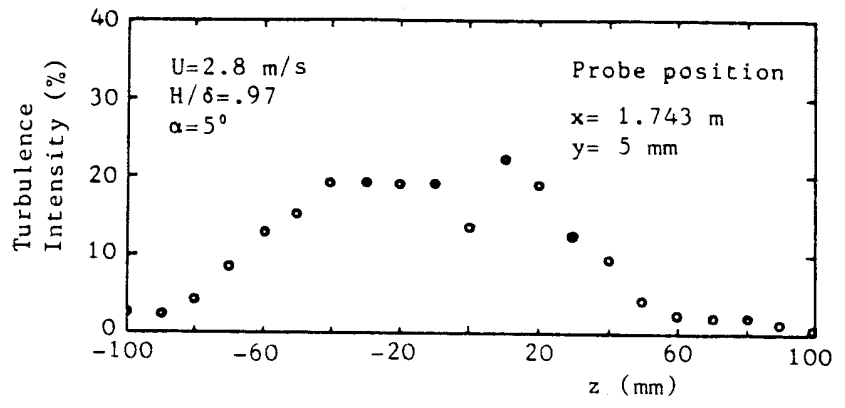

Fig. 8 Spanwise variation of streamwise turbulence intensity

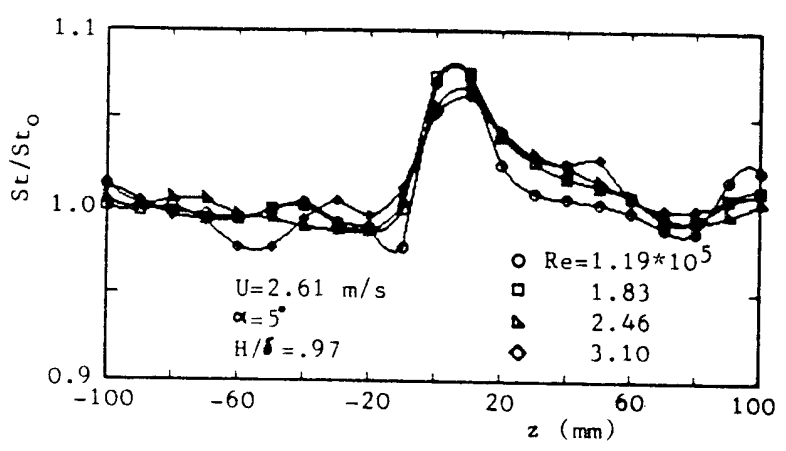

Fig. 9 Spanwise distribution of Stanton number ratio for $U=2.61 \mathrm{~m} / \mathrm{s}$ 
enabled us to elucidate the influence of vortices produced inside (internal vortices: $H / \delta \leq 1$ ) and outside (external vortices: $H / \delta>1$ ) the boundary layer. Figure 10 shows the heat transfer results for the six cases; here, the shift of the enhanced region grows visibly as the height is increased. However, the peak Stanton number ratio does not change with the variation of the height. From Fig. 10 and the previous figures, it is possible to say that the internal and external vortices affect heat transfer in different ways. Internal longitudinal vortices influence only the position of transition so that the symmetrical spreading of the turbulent wedge occurs independent of the vortical motion. On the other hand, external vortices determine not only the transition position but also the development pattern of the transition to a turbulent boundary layer.

\section{Discussion}

\subsection{The effects of the vortical motion and transi- tion on heat transfer}

The introduction of longitudinal vortices in the otherwise laminar boundary layer resulted in large improvements of the heat transfer coefficient. However, this enhancement was much more related to the onset of transition due to the vortex system and the consequent growing of the effective thermal diffusivity than to the vortical motion itself. This result is in agreement with those of similar investigations for Taylor-Görtler and horseshoe vortices which showed a small influence of vortical motion on turbulence-driven heat transfer near the transition region.

Generally speaking, the paek Stanton number

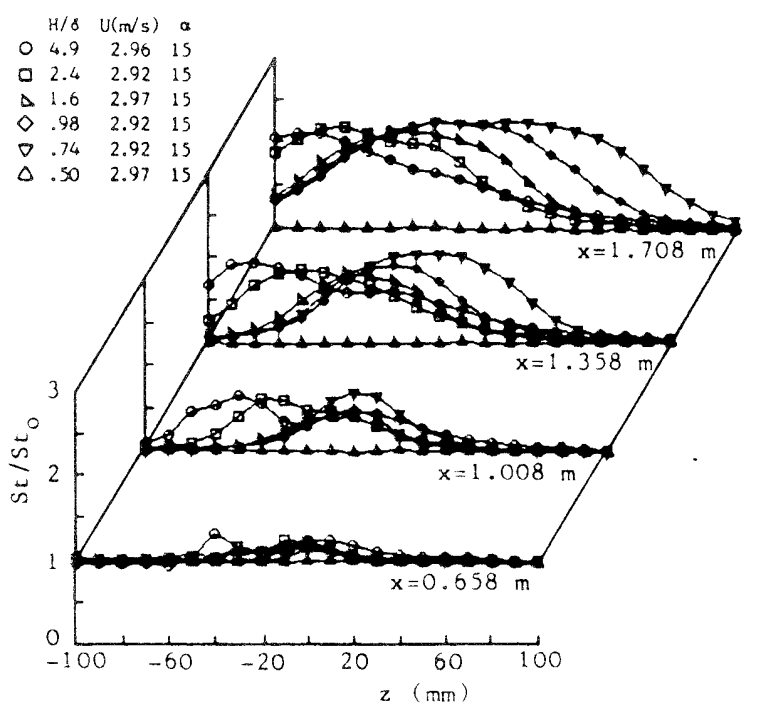

Fig. 10 Axial development of spanwise Stanton number ratio distribution (parameter: height) ratio shifted its spanwise position from the centerline as the transition took place. In Fig. 6 , at $U=2.80 \mathrm{~m} / \mathrm{s}$, the onset of transition can be observed in the fourth row. The position corresponding to negative $z$ (upwash region of the vortex) shows lower Stanton number ratios for the first, second and third rows but a higher one for the fourth. This means that the transition was initiated at the upwash side of the vortex, which is reasonable considering the thickening of boundary layer and the increasing of instabilities that occurred in this region. This observation agrees well with the turbulence intensity measurements (Fig. 8) and the results of Westphal et al. ${ }^{(12)}$, who show that the turbulence intensity in the upwash region of longitudinal vortices embedded in turbulent boundary layers is higher than that in the center or downwash regions.

The above-mentioned feature is also apparent in other cases and can be observed clearly in Figs. 12 and 13. These figures show the axial variation of the relative shift $(s h)$ which was defined as the shift $(e)$ of the Stanton number ratio distribution from the conterline normalized by the span $(b)$ of the region with heat transfer improvement larger than 50 percent of the local peak Stanton value (Fig. 11). The parameter relative shift was introduced to account for the fact that the influence of the absolute shift

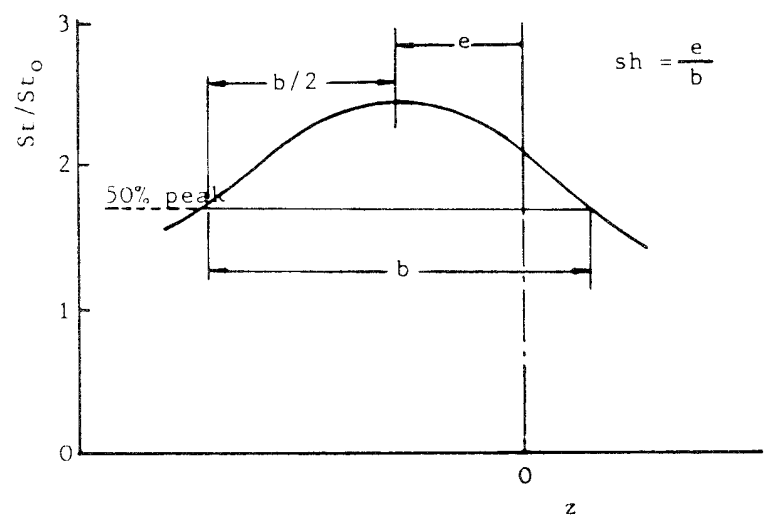

Fig. 11 Definition of relative shift

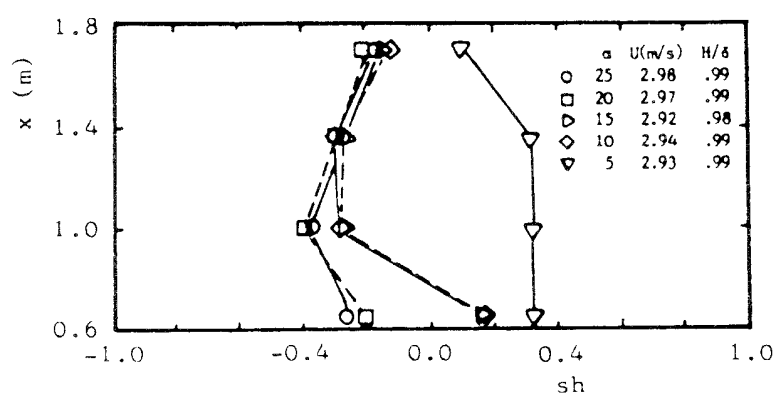

Fig. 12 Axial variation of relative shift for $H / \delta=0.99$ and $\alpha$ from $5^{\circ}$ to $25^{\circ}$ 


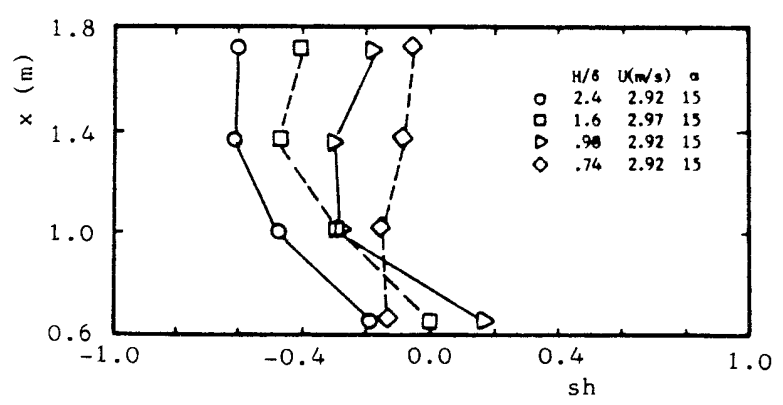

Fig. 13 Axial variation of relative shift for $\alpha=15^{\circ}$ and $H / \delta$ from 0.74 to 2.4

becomes less important when the spreading of the turbulent region is large. Figure 12 shows the axial variation of the relative shift for $H / \delta=0.99$ and five angles of attack. From this figure, it can be observed that the relative shift shows positive values for regimes in which transition does not occur $\left(\alpha=5^{\circ}\right.$, first row of $\alpha=10^{\circ}$ and $\alpha=15^{\circ}$ ). The considerably large positive values of the relative shift for these cases are due to the small values of $b$. For higher values of $\alpha$, the negative relative shift ( 1 ) increases in the second row because the turbulent wedge develops in the upwash side of the vortex, and (2) decreases as the flow moves downstream due to the symmetrical spreading of the turbulent wedge (larger $b$ ). It is also interesting to note that, once transition occurs, the behavior of the "sh" curves for the various angles of attack agrees, even quantitatively.

\section{2 The effects of the external and internal lon- gitudinal vortices on heat transfer}

For half-delta wings with $H / \delta>1$, a longitudinal vortex is formed outside the boundary layer. It is possible to say that a higher $H / \delta$ generates stronger longitudinal vortices. From the heat transfer experiments (Fig. 10), it was found that this kind of vortex produces a distribution of the enhanced heat transfer coefficient throughout the plate that is different from that observed in the cases where $H / \delta \leq 1$. The relative shift grows with the increase of $H / \delta$, as observed in Fig. 13. For higher values of $H / \delta$, the relative shift remains high even far downstream. Although nothing conclusive can be said on the basis of the heat transfer data alone, it seems that the strong external vortex produces a more accentuated influence on heat transfer, even after the transition takes place. For $H / \delta \leq$ 1 , it was observed that the vortices' shifts were relatively small and that their influence on heat transfer was surpassed by the onset of transition. In other words, the internal vortices enhanced heat transfer by inducing transition but, once started, the transition to turbulence developed independent of the vortical motion. This different behavior of the external vor- tices can be explained if we consider that (1) the external longitudinal vortex was stronger than the internal one, (2) the inviscid free stream allowed the vortex to show persistency far downstream, and ( 3 ) the presence of a flat plane induces larger shifts for the stronger vortices in inviscid flows.

\section{Conclusions}

The effect of longitudinal vortices "far enough" from a half-delta wing on heat transfer of an otherwise laminar boundary layer was investigated. The influence of the height and the angle of attack of the vortex generator was elucidated. The following is a summary of the important conclusions.

(1) An embedded longitudinal vortex increases the heat transfer rate by up to 140 percent. The heat transfer enhancement is achieved by inducing the transition to a turbulent boundary layer, with a corresponding rise in the effective thermal diffusivity. The 'vortical motion itself does not considerably affect the heat transfer.

(2) Internal and external longitudinal vortices affect heat transfer in different ways. While the internal vortices influence only the position of the transition, the external ones affect also the development pattern of the transition to turbulence.

(3) The onset of the transition to turbulence is mainly dependent on the strength of the vortex, which is closely related to $H / \delta$ and the angle of attack.

(4) The transition is initiated in the upwash region of the vortex, where the instabilities are larger. The consequent spreading of the turbulent region occurs symmetrically for $H / \delta \leq 1$.

(5) Concerning the influence of the vortical motion in the laminar regime, it was found that a rise of the heat transfer coefficient occurs in the downwash region, where the boundary layer is thinner.

\section{Acknowledgments}

We wish to thank Mr. M. Kinukawa and Mr. Y. Nagai for their assistance in constructing the experimental apparatus and performing the experiments. The second author gratefully acknowledges the Ministry of Education of Japan for financial support in the form of a graduate scholarship.

\section{References}

(1) McCormack, P. D., Welker, H. and Kelleher, M., Taylor-Görtler Vortices and Their Effect on Heat Transfer, Trans. ASME, J. Heat Transf., Vol. 92 (1970), p. 101.

(2) Kottke, V., Taylor-Görtler Vortices and Their Effect on Heat and Mass Transfer, Proc. 8th Int. Heat Transf. Conf., Vol. 3 (1986), p. 1139. 
(3) Schuz, G. and Kottke, V., Horseshoe Vortices and Their Effect on Heat and Mass Transfer, Proc. 6th Simp. Turbulent Shear Flows (1987), No. 184.

(4) Edwards, F. J. and Alker, C. J. R., The improvement of Forced Convection Heat Transfer using Surface Protrusions in the Form of (a) Cubes, and (b) Vortex Generators, Proc. 5th Int. Heat Transf. Conf., Vol. 2 (1974), p. 244.

(5) Russel, C. M. B., Jones, T. V., and Gee, G. H., Heat Transfer Enhancement Using Vortex Generators, Proc. 7th Int. Heat Transf. Conf., Vol. 3 (1982), p. 283.

(6) Fiebng, M., Kallweit, P. and Mitra, N. K., Wing Vortex Generators for Heat Transfer Enhancement, Proc. 8th Int. Heat Transf. Conf., Vol. 6 (1986), p. 2909.

(7) Turk, A. K. and Junkhan, G. H., Heat Transfer Enhancement Downstream of Vortex Generators on a Flat Plate, Proc. 8th Int. Heat Transf. Conf., Vol. 6 (1986), p. 2903.

(8) Eibeck, P. and Eaton, J.K., An Experimental Investigation of the Heat Tranfer Effects of a Longitudinal Vortex Embedded in a Turbulent Boundary Layer, Stanford Univ.-Thermosciences Div. - Report MD-48 (1985).

(9) Eibeck, P. and Eaton, J. K., The Effects of Longitudinal Vortices Embedded in a Turbulent Bound. ary Layer on Momentum and Thermal Transport, Proc. 8th Int. Heat Transf. Conf., Vol. 3 (1986), p. 1115.

(10) Eibeck, P. and Eaton, J. K., Heat Transfer Effects of a Longitudinal Vortex Embedded in a Tumbulent Boundary Layer, Trans., ASME, J. Heat Transf., Vol. 109 (1987), p. 16.

(11) Shabaka, I. M. M. A., Mehta, R. D. and Bradshaw, P., Longitudinal Vortices Embedded in Turbulent Boundary Layer. Part 1. Single Vortex, J. Fluid Mech., Vol. 155 (1985), p. 37.

(12) Westphal, R. V., Eaton, J. K. and Pauley, W. R. Interaction Between a Vortex and a Turbulent Boundary Layer in a Stremwise Pressure Gradi. ent, Turbulent Shear Flows 5 (1987), p. 266, Springer-Verlag.

(13) Mehta, R. D. and Cantwell, E., Properties of a Half-Delta Wing Vortex, Proc. 6th Symp. Turbulent Shear Flows (1987), No. 8-3.

(14) Pauley, W. R. and Eaton, J. K., The Effect of Embedded Longitudinal Vortex Pairs on Turbulent Boundary Layer Heat Transfer, Proc. 2th Int. Symp. on Transp. Phenom. in Turb. Flows (1987), p. 269.

(15) ANSI/ASME PTC 19.1, Measurement Uncertainty (1985)

(16) Rubesin, M. W., Inouye, M. and Parikh, P.G., Forced Convection-External Flows, Handbook of Heat Transf-Fund. (1985), McGraw-Hill.

(17) Tani, I., Komoda, H. and Komatsu, Y., Univ. of Tokyo-Aero. Res. Inst., Rep. No. 375 (1962). 